\title{
Caracterização por Reconstrução Tridimensional da Superfície de Fratura Gerada por Fadiga em Soldas Subaquáticas Molhadas
}

\author{
Natália Maia Fonseca ${ }^{1}$, Paulo José Modenesi ${ }^{1}$, Ariel Rodriguez Arias ${ }^{2}$ \\ 1 Universidade Federal de Minas Gerais - UFMG, Departamento de Engenharia Metalúrgica e de Materiais, Belo Horizonte, MG, Brasil. \\ 2 Universidade Federal de Minas Gerais - UFMG, Departamento de Engenharia Mecânica, Belo Horizonte, MG, Brasil.
}

Recebido: 17 Jan., 2018

Aceito: 15 Maio, 2018

E-mails: nataliamaiafonseca@gmail. com (NMF), modenesi@ufmg.br (PJM), arielra2506@gmail.com (ARA)
Resumo: Superfícies de fratura geradas por fadiga em soldas subaquáticas molhadas foram caracterizadas por reconstruções tridimensionais usando estereofotogrametria em um microscópio eletrônico de varredura. Análise de perfil, rugosidade e dimensão fractal foram avaliadas para essas superfícies com o intuito de correlacioná-los com os resultados de ensaios de propagação de trinca e densidade de porosidade. Verificou-se que o parâmetro de rugosidade superficial Sq apresentou-se como mais eficiente para descrever e comparar as superfícies estudadas do que a dimensão fractal. Além disso, pôde-se confirmar a aplicabilidade da técnica usada e da metodologia desenvolvida para o estudo proposto com a ressalva de que um estudo mais aprofundado é necessário para compreender os mecanismos de propagação de trinca por fadiga em soldas subaquáticas molhadas.

Palavras-chave: Superfície de fratura; Fadiga; Soldagem subaquática molhada; Reconstrução tridimensional.

\section{Characterization by Three-Dimensional Reconstruction of the Fatigue Fracture Surface in Underwater Wet Welds}

\begin{abstract}
Fatigue fracture surfaces in wet underwater welds were characterized by means of three-dimensional reconstructions using stereophotogrammetry in a scanning electron microscope. Profile analysis, roughness and fractal dimension were evaluated for these surfaces in order to compare them with the results of crack growth tests and welding depth. It could be seen the areal roughness parameter $\mathrm{Sq}$ as more effective than the fractal dimension to describe and compare the studied surfaces. In addition, it was possible to endorse the pertinence of the used technique and the developed methodology for the proposed study. Nevertheless, it is stated that further study is required in order to fully understand the mechanisms of fatigue crack propagation in wet underwater welds.
\end{abstract}

Key-words: Fracture surface; Fatigue; Underwater wet weld; Three-dimensional reconstruction.

\section{Introdução}

Atividades como a exploração de óleo e gás offshore tornaram necessária a soldagem de estruturas e componentes dentro da água, principalmente para reparo e manutenção. Por sofrerem ações de solicitações mecânicas variáveis, devido ao movimento das ondas e correntes oceânicas, essas instalações estão sujeitas a sofrerem falha por fadiga e regiões como cordão de solda e zona termicamente afetada são mais susceptíveis.

As soldas subaquáticas molhadas, mais especificamente, são caracterizadas por grandes proporções de descontinuidades (poros principalmente) inerentes ao processo de soldagem devido à absorção de gases pelo metal líquido e à alta taxa de resfriamento a que a poça de fusão fica submetida nesse tipo de ambiente [1].

A porosidade pode ter efeito negativo nas propriedades mecânicas dos materiais e para o comportamento por fadiga em particular as trincas podem iniciar rapidamente nos poros e a vida em fadiga fica, então, dominada pela fase de propagação da trinca, que, por sua vez, também é influenciada pela porosidade. Nesse sentido, a presença de porosidade causa redução da vida em fadiga [2-6] e também provoca considerável dispersão de sua duração [5]. Além disso, as características da porosidade como a aleatoriedade na distribuição, tamanho, quantidade, forma e conectividade e distância entre poros 
adjacentes tornam o estudo da fratura de materiais porosos mais difícil de ser estudado [4,7]. Como a porosidade pode ser um fator para a redução das propriedades mecânicas de um material, o estudo do comportamento por fadiga de soldas subaquáticas molhadas vem aumentando [8,9].

Arias e Bracarense [9] e Arias [10], em seu estudo sobre propagação de trincas por fadiga em soldas subaquáticas molhadas, observou que, com o aumento da porosidade na solda, a taxa de propagação é reduzida em ensaios de fadiga com valores de $\Delta K$ (amplitude do fator de intensidade de tensões) baixos a intermediários (14 a $26 \mathrm{MPa} \cdot \mathrm{m}^{1 / 2}$ ). A influência da porosidade é negativa para valores maiores de $\Delta K$ em que se observa redução da resistência à fadiga. No primeiro caso, o autor propôs que tanto a ramificação e deflexão da trinca pela sua interação com poros, quanto à propagação simultânea de trincas secundárias e o fechamento da trinca induzido por rugosidade sejam fatores que expliquem esse comportamento. Já para o caso de $\Delta \mathrm{K}$ elevado ( 26 a $30 \mathrm{MPa} \cdot \mathrm{m}^{1 / 2}$ ), foi proposto que a porosidade causa o aumento da tensão local na ponta da trinca, explicando assim o aumento da taxa de propagação observado.

Matlock et al. [11] também chegaram à conclusão similar sobre a propagação de trincas por fadiga em soldas subaquáticas molhadas. Os resultados deste trabalho indicam uma taxa de propagação da trinca por fadiga menor do que de um material similar sem porosidade para valores de $\Delta \mathrm{K}$ inferiores a aproximadamente $30 \mathrm{MPa} \cdot \mathrm{m}^{1 / 2}$. Este efeito foi atribuído ao ancoramento da ponta da trinca pelos poros pela redução do fator de intensidade de tensão. Por outro lado, quando os valores de $\Delta K$ são mais elevados a tensão local na ponta da trinca aumenta pela presença da porosidade. Este efeito facilitaria a nucleação e crescimento de trincas entre poros acelerando a velocidade global de crescimento da trinca.

A maior parte dos estudos feitos para investigar os danos causados por porosidade em materiais submetidos à fadiga é baseada em observações das superfícies de fratura realizadas via microscópio óptico e microscópio eletrônico de varredura (MEV). As informações obtidas a partir da análise da superfície de fratura são usadas para avaliar as causas da falha e os fatores que a influenciam, podendo ser usadas para então prevenir futuras falhas.

Nas últimas décadas, técnicas mais avançadas envolvendo caracterização tridimensional, como a estereofotogrametria [12-14], têm sido empregadas para se entender os mecanismos de fratura de materiais dúcteis e também as características dos danos causados por fadiga.

O princípio da estereofotogrametria [15] é baseado na obtenção de um par ou trio estereoscópico de imagens. Essas imagens são obtidas normalmente por meio do MEV de uma mesma região da amostra sob perspectivas diferentes, o que é alcançado pela inclinação eucêntrica da amostra. Os pontos correspondentes nessas imagens são detectados por algoritmos e as alturas desses pontos são determinadas pelas disparidades (diferença de localização de uma determinada característica) observadas nas imagens. Além da obtenção do modelo tridimensional da superfície estudada, softwares comerciais baseados neste princípio também permitem a obtenção de seus perfis topográficos, parâmetros de rugosidade e também a dimensão fractal (Df), que podem auxiliar no estudo dessas superfícies.

Gadelmawla et al. [16] e Blunt e Jiang [17] fazem uma descrição detalhada de parâmetros de rugosidade de perfil e de área, respectivamente, usados para análise de superfícies. Os parâmetros de perfil são mais disseminados e empregados por já serem normatizados desde a década de 1980 pelas normas ISO 4287 e 4288 , em contrapartida aos parâmetros tridimensionais que tiveram seu desenvolvimento a partir de 1998 no projeto europeu Surfstand [18] e só foram completamente padronizados na ISO 25178 em 2012. Apesar disso, as medições bidimensionais fornecem uma descrição incompleta da topografia real da superfície analisada. As informações obtidas via medições de área, por outro lado, são mais abrangentes que as de perfil [19] pelo fato de ser usado um maior número de pontos de medidas.

Entretanto, medidas diretas de rugosidade não apresentam independência quanto à escala, o que faz com que a dimensão fractal esteja sendo muito pesquisada e empregada como parâmetro dimensional na caracterização de topografias [20]. Superfícies rugosas podem possuir uma propriedade que permite que a informação de rugosidade em todas as escalas seja mantida e que, se ampliada repetidamente, o aumento de detalhes da rugosidade é observado até a nanoescala [20]. Esse comportamento é descrito pela geometria fractal que é capaz de caracterizar a rugosidade da superfície por parâmetros independentes da escala, fornecendo informações sobre rugosidade em todas as escalas de comprimento que exibem o comportamento fractal. A dimensão fractal, por sua vez, é caracterizada por números não inteiros, que indicam o grau de complexidade e irregularidade do fractal. A geometria do fractal é considerada como a transição entre duas geometrias regulares. Dessa forma, uma superfície fractal, por exemplo, tem dimensão entre um plano e um volume, $2<\mathrm{Df}<3$ [21-23]. 
Embora desenvolvidas nas últimas décadas, programas de reconstrução de superfícies a partir de imagens estereoscópicas são ainda aplicados de forma incipiente na caracterização de superfícies de fratura, particularmente de soldas. Nesse sentido, esse trabalho tem como foco estudar a aplicação da estereofotogrametria como técnica de caracterização da topografia de superfícies no estudo da superfície de fratura de corpos de prova de solda subaquática submetidos a ensaio de fadiga visando complementar informações relativas à propagação das trincas.

\section{Materiais e Métodos}

\subsection{Corpos de prova}

Os corpos de prova do tipo $C(T)$ de ensaios de fadiga de solda subaquática são provenientes de outro estudo realizado no Laboratório de Robótica, Soldagem e Simulação da UFMG [8-10]. O metal base (aço ASTM A36) foi soldado com eletrodo rutílico (AWS E6013) dentro de uma câmera hiperbárica, simulando pressões hidrostáticas de até $90 \mathrm{~m}$ e utilizando um dispositivo mecanizado para soldagem em conjunto com uma fonte projetada para soldagem subaquática.

Estes corpos de prova foram ensaiados por dois métodos para a determinação da taxa de crescimento de trincas por fadiga adotados por Arias [10] e padronizados pela norma ASTM E647-15, sendo método de amplitude de carga constante ( $\Delta \mathrm{K}$ crescente) para estudo da propagação de trinca na região II e método de amplitude de carga constante ( $\Delta K$ decrescente) objetivando-se esse estudo próximo do limiar (região I).

Para o primeiro método, em que as cargas máxima e mínima são mantidas constantes com uma variação senoidal entre elas, foi aplicada a frequência de $30 \mathrm{~Hz}$ para taxas de propagação menores que $10^{-5} \mathrm{~mm} /$ ciclo, $15 \mathrm{~Hz}$ para taxas entre $10^{-5} \mathrm{e} 10^{-4} \mathrm{~mm} / \mathrm{ciclo}, 5 \mathrm{~Hz}$ para taxas maiores $10^{-4} \mathrm{~mm} /$ ciclo. A razão de carregamento aplicada foi de 0,1 e amplitude de carga de $10 \mathrm{kN}$. Já no método de amplitude de carga decrescente, uma frequência de $30 \mathrm{~Hz}$ foi usada até a obtenção de taxas de crescimento próximas a $10^{-7} \mathrm{~mm} /$ ciclo com razão de carregamento de 0,1 também. A medição do comprimento da trinca foi conduzida pela técnica de flexibilidade elástica por meio de um extensômetro (clip-gage) localizado na boca do entalhe.

Quatro corpos de prova de ensaios de fadiga de solda subaquática (Figura 1) foram selecionados de forma a se poder estudar os efeitos da profundidade $(10$ e $90 \mathrm{~m})$ como condição de soldagem, obtendo-se diferentes populações de porosidade e da solicitação como resultado do método de ensaio, conforme descrito na Tabela 1.

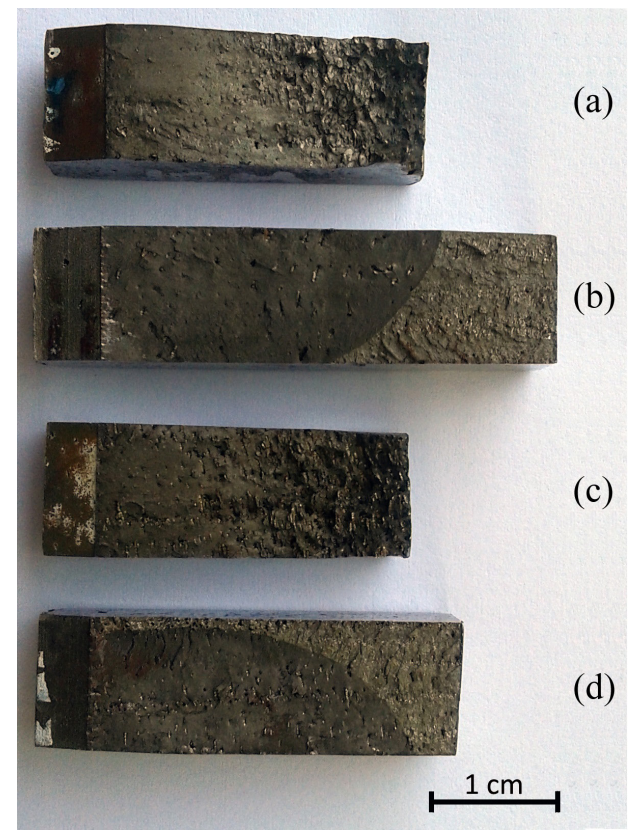

Figura 1. Corpos de prova de fadiga de solda subaquática selecionados: (a) CC10; (b) CD10; (c) CC90; e (d) CD90. 
Tabela 1. Corpos de prova selecionados.

\begin{tabular}{ccc}
\hline Corpo de prova & Profundidade de soldagem [m] & Método de ensaio \\
CC10 & 10 & Amplitude de carga constante \\
CD10 & 10 & Amplitude de carga decrescente \\
CC90 & 90 & Amplitude de carga constante \\
CD90 & 90 & Amplitude de carga decrescente \\
\hline
\end{tabular}

Nos corpos de prova ensaiados pelo método de redução de carga (CD10 e CD90), as trincas não propagaram de forma simétrica, conforme visto na Figura 1, devido à própria condição do teste. É reportado [24] que valores mais elevados de $\Delta K$ contribuem para a redução da assimetria da propagação das trincas, razão essa que pode explicar a não ocorrência de assimetria nas amostras CC10 e CC90.

\subsection{Mapeamento dos corpos de prova}

Inicialmente, realizou-se a caracterização de toda a superfície das amostras em estudo usando microscopia óptica de baixa ampliação com um microscópio estereográfico. Macrografias com ampliação de 4x foram obtidas das superfícies de fratura, resultando em cerca de 60 imagens para cada corpo de prova. Essas imagens foram então unidas em um software de edição de imagens.

Algumas regiões de interesse em cada amostra foram selecionadas para serem submetidas à reconstrução por estereofotogrametria com base nas condições do ensaio de propagação de trincas realizado por Arias [10]. Valores de $\Delta \mathrm{K}$ foram mapeados a partir das curvas $\log (\mathrm{da} / \mathrm{dN}) \mathrm{vs}$. $\log \Delta \mathrm{K}$ e as regiões para análise foram escolhidas com base em valores semelhantes deste parâmetro para os diferentes corpos de prova com o intuito de se realizar posterior comparação entre os resultados, conforme ilustra a Figura 2.

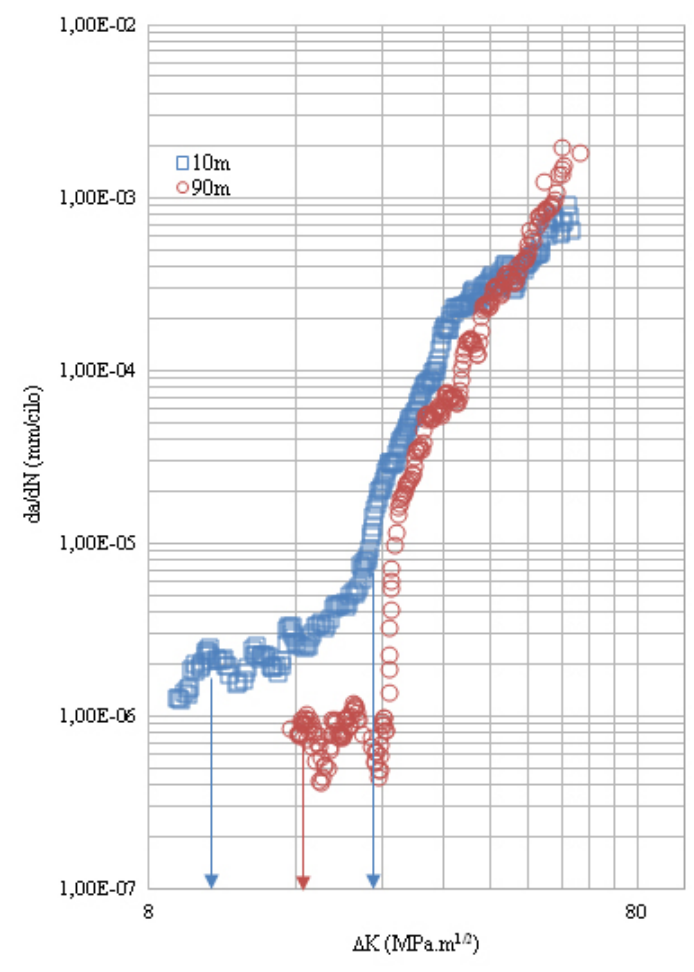

Figura 2. Curva $\log \Delta \mathrm{K}$ vs. $\log (\mathrm{da} / \mathrm{dN})$ de soldas produzidas a 10 e $90 \mathrm{~m}$ de profundidade e ensaiadas pelo método de amplitude de carga decrescente, mostrando as regiões analisadas. 
Caracterização por Reconstrução Tridimensional da Superfície de Fratura Gerada por Fadiga em Soldas Subaquáticas Molhadas

A Tabela 2 mostra os valores de $\Delta \mathrm{K}$, mapeados e selecionados a partir das curvas $\log \Delta \mathrm{K} v \mathrm{~s}$. $\log (\mathrm{da} / \mathrm{dN})$, ao longo dos corpos de prova. Em cada posição, duas regiões a $4 \mathrm{~mm}$ da borda superior (a) e a $4 \mathrm{~mm}$ da borda inferior (b) do corpo de prova foram avaliadas.

Tabela 2. Valores de $\Delta \mathrm{K}$ das posições selecionadas para análise.

\begin{tabular}{|c|c|c|c|c|}
\hline & Regiões & CC10/20a e b & $\mathrm{CC} 10 / 32 \mathrm{a}$ e $\mathrm{b}$ & CC10/41a e b \\
\hline \multirow[t]{3}{*}{ CC10 } & $\mathrm{a}[\mathrm{mm}]$ & 4,1 & 12,0 & 16,0 \\
\hline & $\Delta \mathrm{K}\left[\mathrm{MPa} \cdot \mathrm{m}^{1 / 2}\right]$ & 21,1 & 32,7 & 41,5 \\
\hline & Regiões & CD10/25a e b & CD10/12a e b & \\
\hline \multirow[t]{3}{*}{ CD10 } & $\mathrm{a}[\mathrm{mm}]$ & 5,8 & 13,8 & - \\
\hline & $\Delta \mathrm{K}\left[\mathrm{MPa} \cdot \mathrm{m}^{1 / 2}\right]$ & 24,0 & 10,5 & \\
\hline & Regiões & $\mathrm{CC} 90 / 20 \mathrm{a}$ e $\mathrm{b}$ & $\mathrm{CC} 90 / 32 \mathrm{a}$ e b & CC90/41a e b \\
\hline \multirow[t]{3}{*}{ CC90 } & $\mathrm{a}[\mathrm{mm}]$ & 3,4 & 11,4 & 15,4 \\
\hline & $\Delta \mathrm{K}\left[\mathrm{MPa} \cdot \mathrm{m}^{1 / 2}\right]$ & 20,2 & 32,6 & 41,3 \\
\hline & Regiões & CD90/25a e b & CD90/12a e b & \\
\hline \multirow[t]{2}{*}{ CD90 } & $\mathrm{a}[\mathrm{mm}]$ & 2,7 & 10,7 & - \\
\hline & $\Delta \mathrm{K}\left[\mathrm{MPa} \cdot \mathrm{m}^{1 / 2}\right]$ & 26,0 & 14,4 & \\
\hline
\end{tabular}

\subsection{Coleta dos pares estereoscópicos}

Após a seleção das áreas de interesse, trios de imagens estereoscópicas foram obtidos por meio do microscópio eletrônico de varredura FEI Quanta 3D FEG a partir da análise dos sinais de elétrons secundários. Todos os trabalhos envolvendo microscopia eletrônica foram conduzidos no Centro de Microscopia da UFMG.

A inclinação da amostra no eixo horizontal deve ser realizada de forma eucêntrica, resultando em um ponto central estático na imagem, condição esta necessária para a precisão da reconstrução. A qualidade das imagens traduzida em brilho, contraste e nitidez também é essencial para o correto cálculo das informações 3D. A ampliação foi selecionada de forma a se obter informações relevantes na imagem, objetivando avaliar a interação trinca-poro. Sendo assim, foram coletadas imagens com 120 e 130x de ampliação. Já o ângulo de inclinação deve ser selecionado de forma a garantir que as informações das micrografias possam ser devidamente interpretadas pelo software, neste caso, foram utilizados trios de imagens com $-5,0$ e $+5^{\circ}$ de inclinação.

Essa inclinação foi selecionada após testes com os valores de $\pm 3, \pm 5, \pm 7$ e $\pm 10^{\circ}$, que são comumente reportados na literatura $[12-14,25,26]$. Verificou-se que os modelos reconstruídos com o trio $\pm 5^{\circ}$ representaram de forma mais fiel às características topográficas quando comparadas às imagens obtidas pelo MEV e também os valores de rugosidades da superfície que tiveram menores diferenças em relação aos obtidos pela técnica de perfilometria de contato.

A quantidade de porosidade nessas imagens foi determinada com o software de processamento de imagens ImageJ. A área total reconstruída foi medida e também foi determinada a área total de poros, obtendo-se assim a porcentagem de porosidade para cada região analisada.

\subsection{Reconstruções das superfícies de fratura}

A construção dos modelos tridimensionais foi feita pelo software MeX [27] da Alicona Imaging $\mathrm{GmbH}$ utilizando um trio de imagens estereoscópico. Com a inclusão de mais uma imagem além das inclinadas positiva e negativamente com relação ao eixo x, os ângulos são calibrados durante o processamento das imagens pelo software e assim não há preocupação quanto à precisão dos ângulos fornecidos pelo MEV.

O primeiro passo para a reconstrução é a entrada das imagens e de parâmetros como o ângulo de inclinação e a escala de ampliação. Em seguida, deve-se configurar valores manual ou automaticamente para o Global Offset, que representa o ponto de partida para o algoritmo do MeX corresponder pontos nas imagens do trio baseado nas disparidades. Por último, uma região de interesse na imagem onde a reconstrução 3D será feita é selecionada. 
É possível, então, remover textos e partes da imagem consideradas desnecessárias. Após a obtenção dos modelos tridimensionais, algumas operações podem se fazer necessárias para ajustar a superfície criada. Tais operações podem ser feitas no próprio MeX que fornece diversas ferramentas para correção dos modelos, como remoção de defeitos na superfície e de parte do conjunto de dados, por exemplo. No caso deste trabalho, a ferramenta Workpiece Coordinate System foi empregada a fim de se ajustar o plano do modelo reconstruído.

As análises que foram realizadas pelo MeX neste trabalho foram partir do módulo ProfileRoughnessMeasurement a extração de perfil, cálculo de parâmetros de rugosidade ( $\mathrm{Ra}, \mathrm{Rq}, \mathrm{Rz}$ ) de acordo com as normas ISO 4287/4288, bem como a filtragem de perfis por meio do valor $\lambda c$ (filtra os comprimentos de onda acima ou abaixo do comprimento de cut-off adotado, separando ondulação de rugosidade) de acordo com ISO 11562 e análise de área que fornece a determinação dos parâmetros Sa, Sq e Sz e o cálculo da dimensão fractal (ISO 25178-2 e ASME B46.1) pelo módulo SurfaceTextureMeasurement.

Para as medições de rugosidade 2D foi utilizado comprimento de análise de cerca de 1,6 mm (comprimento do perfil primário), o que resultou em comprimentos do perfil de rugosidade de 1,3 $\mathrm{mm}$, já que $10 \%$ do perfil primário do início e do fim não são incluídos nos perfis filtrados. Já para as medições de rugosidade 3D, a área avaliada foi de 2,3 × 2,1 mm. O cut-off de $800 \mu \mathrm{m}$, limitado pelo comprimento de avaliação, foi selecionado para eliminar a influência das ondulações nas análises.

Paralelamente, análises de algumas dessas áreas foram conduzidas por perfilometria de contato, com o intuito de verificar a reprodutibilidade das reconstruções obtidas pelo MeX com respeito a coordenada $z$. Os dados obtidos pelo perfilômetro foram tratados em um software integrado para a obtenção das superfícies tridimensionais e perfis topográficos.

Além disso, com o intuito de verificar a reprodutibilidade das reconstruções obtidas pelo MeX com respeito à coordenada $z$, foi realizada a reconstrução de uma região de uma amostra com irregularidades superficiais conhecidas e com diferentes profundidades de sulcos para testar diferentes condições de aquisição de imagem e de seu processamento. Para isso, primeiramente uma amostra de níquel microusinada, com sulcos de 30 e 50 micrômetros de profundidade, teve uma área de $2 \times 3 \mathrm{~mm}$ analisada por meio do perfilômetro de contato e, em seguida, foi realizada a reconstrução no MeX a partir das imagens obtidas no MEV da mesma área.

\section{Resultados e Discussão}

\subsection{Características gerais das superfícies de fratura}

Exemplos das imagens da superfície de fratura coletadas via MEV das regiões de interesse para a reconstrução no software MeX são mostradas na Figura 3 e na Figura 4. As imagens das amostras CD possuem aparência semelhante a essas.

É possível verificar que o aumento da pressão hidrostática na soldagem subaquática molhada acarreta em aumento da porosidade, conforme relatado por diversos autores [10,28]. As amostras soldadas a $90 \mathrm{~m}$ de profundidade (CC90 e CD90) apresentam, de maneira geral, maior rede de poros nas superfícies de fratura do que as amostras obtidas a $10 \mathrm{~m}$ de profundidade (CC10 e CD10). Apesar disso, como apenas algumas regiões das superfícies dos corpos de prova selecionadas foram avaliadas, algumas regiões dos corpos de prova CC10 e CD10 apresentam maior porosidade que algumas dos corpos de prova CC90 e CD90 (Tabela 3). A partir da observação dessas superfícies é também possível verificar que esses poros não se distribuem de forma uniforme e também não possuem uniformidade de tamanho e forma.

Além disso, observa-se que a topografia da superfície de fratura é mais acidentada para as soldas com quantidade maior de poros devido à interação entre a trinca e estes. De acordo com os estudos de Arias [10], essa interação é mais acentuada para valores mais elevados de $\Delta K$. Ainda segundo este pesquisador, ocorrem ramificações ou trincas secundárias ao longo do caminho da trinca, sendo este processo mais acentuado para soldas produzidas a maior profundidade, consequência também das interações entre trincas e poros. 

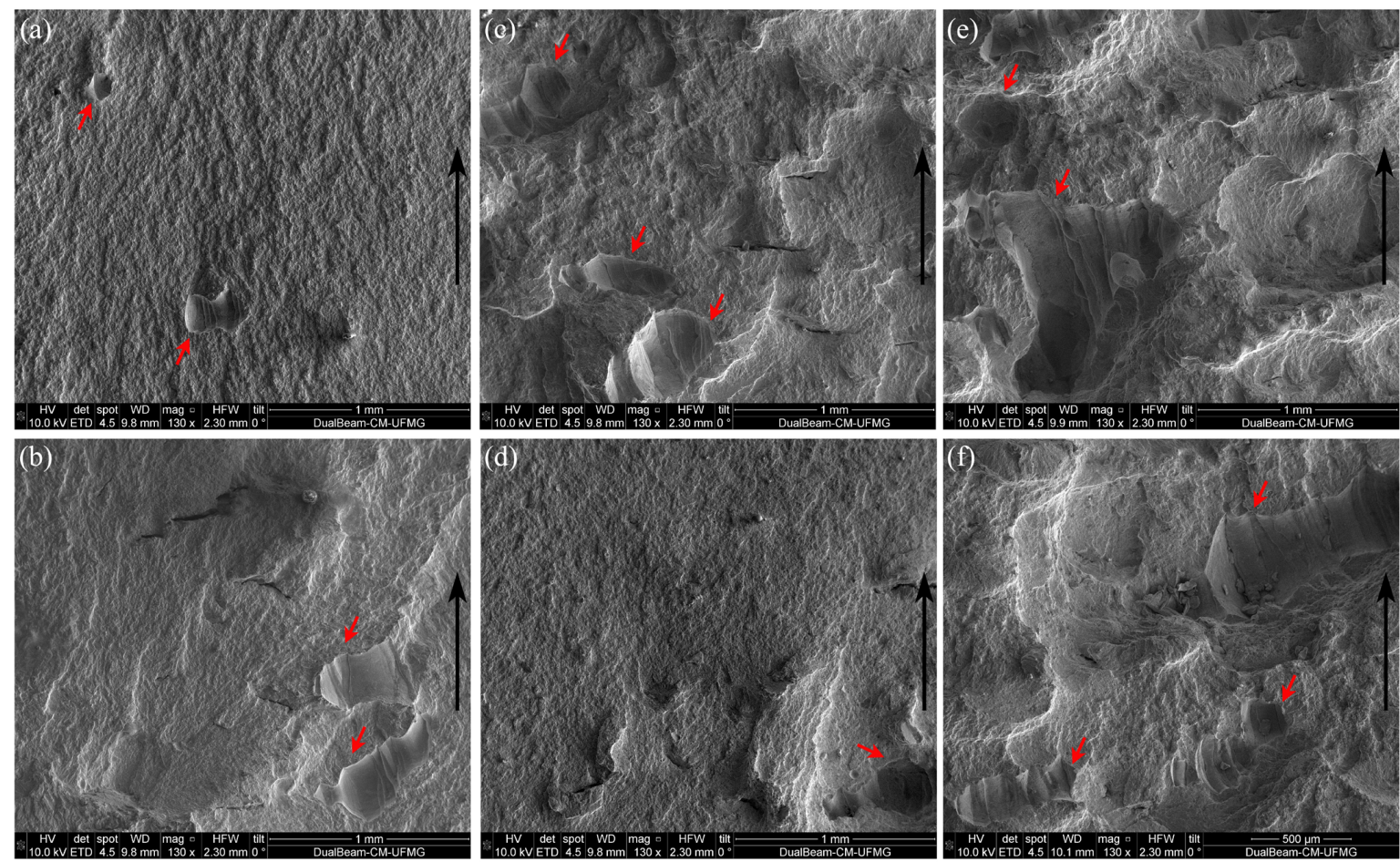

Figura 3. Imagens da superfície de fratura obtidas por MEV do corpo de prova $\mathrm{CC} 10$ nas posições selecionadas: (a) CC10/20a; (b) CC10/20b; (c) CC10/32a; (d) CC10/32b; (e) CC10/41a; (f) CC10/41b. A direção da propagação da trinca e os poros estão indicados.
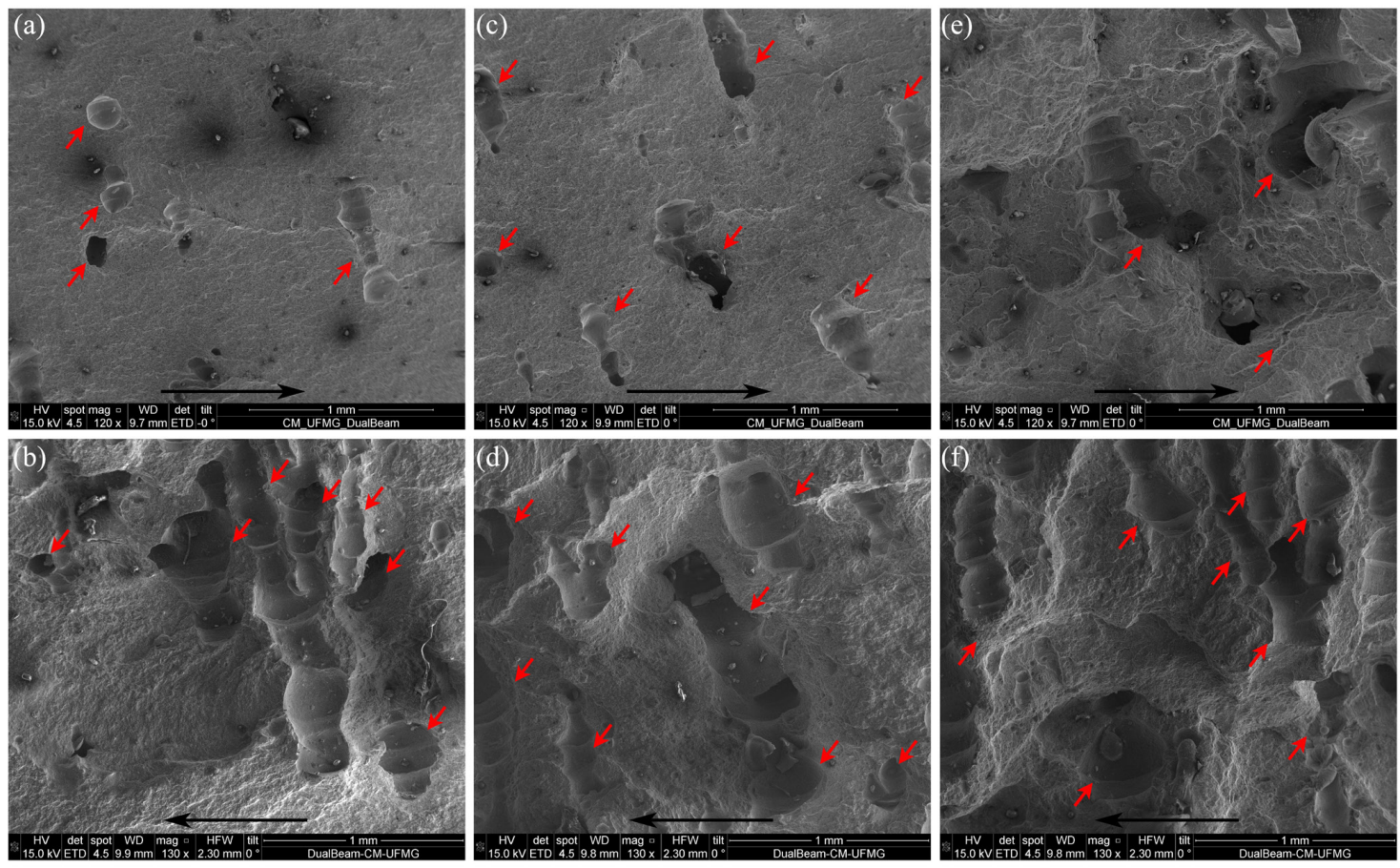

Figura 4. Imagens da superfície de fratura obtidas por MEV do corpo de prova CC90 nas posições selecionadas: (a) CC90/20a; (b) CC90/20b; (c) CC90/32a; (d) CC90/32b; (e) CC90/41a; (f) CC90/41b. A direção da propagação da trinca e os poros estão indicados. 
Tabela 3. Porcentagem de poros nas regiões das superfícies de fratura avaliadas.

\begin{tabular}{cc}
\hline Amostra / Região & Porosidade (\%) \\
CC10/32a & 8,7 \\
CC10/32b & 2,4 \\
CC10/41a & 20,1 \\
CC10/41b & 17,9 \\
CD10/25a & 7,7 \\
CD10/25b & 10,3 \\
CD10/12a & 4,3 \\
CD10/12b & 2,6 \\
CC90/20a & 4,3 \\
CC90/32a & 8,0 \\
CC90/32b & 27,8 \\
CC90/41a & 17,5 \\
CC90/41b & 23,7 \\
CD90/25a & 6,9 \\
CD90/25b & 10,2 \\
CD90/12a & 10,1 \\
CD90/12b & 16,0 \\
\hline
\end{tabular}

\subsection{Comparação com resultados de perfilometria}

A Figura 5 apresenta como exemplo o resultado obtido por meio de um perfilômetro de contato e a Figura 6 o resultado pelo MeX para a região CD10/25a da superfície de fratura mostrada na Figura 7 . Na Tabela 4 são apresentados os valores dos parâmetros de rugosidade obtidos para essa superfície por ambas as técnicas. O cut-off adotado foi de $800 \mu \mathrm{m}$ e os parâmetros de perfil foram obtidos conforme indica a Figura 8.

Verifica-se qualitativamente que os formatos dos perfis topográficos estão semelhantes. De maneira quantitativa, uma diferença de altura (diferença entre a maior e a menor coordenada) de cerca de $12 \%$ foi encontrada para a coordenada $z$.

Com relação aos parâmetros de rugosidade calculados por ambos os métodos, percebe-se boa concordância entre $\mathrm{Ra}$ e $\mathrm{Rq}$, com o parâmetro Ra para o modelo reconstruído com ângulo de inclinação de $\pm 5^{\circ}$ tendo menor diferença (8\%) com relação à perfilometria. Resultado semelhante com relação às diferenças entre os parâmetros de rugosidade obtidos por meio das duas técnicas foi obtido para a análise conduzida com a amostra microusinada.

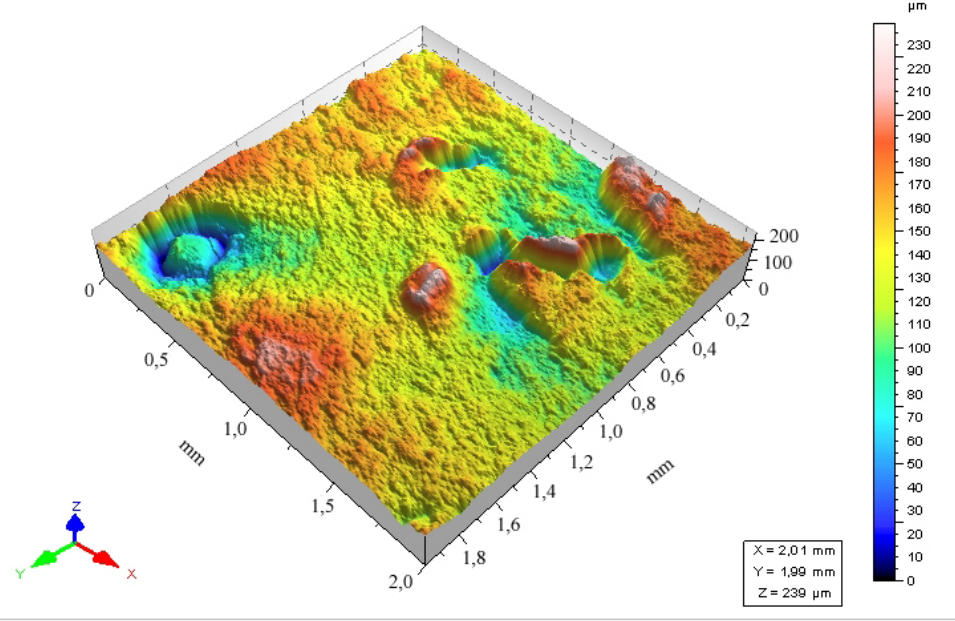

Figura 5. Topografia tridimensional obtida pelo perfilômetro da superfície de fratura da região CD10/25a. 

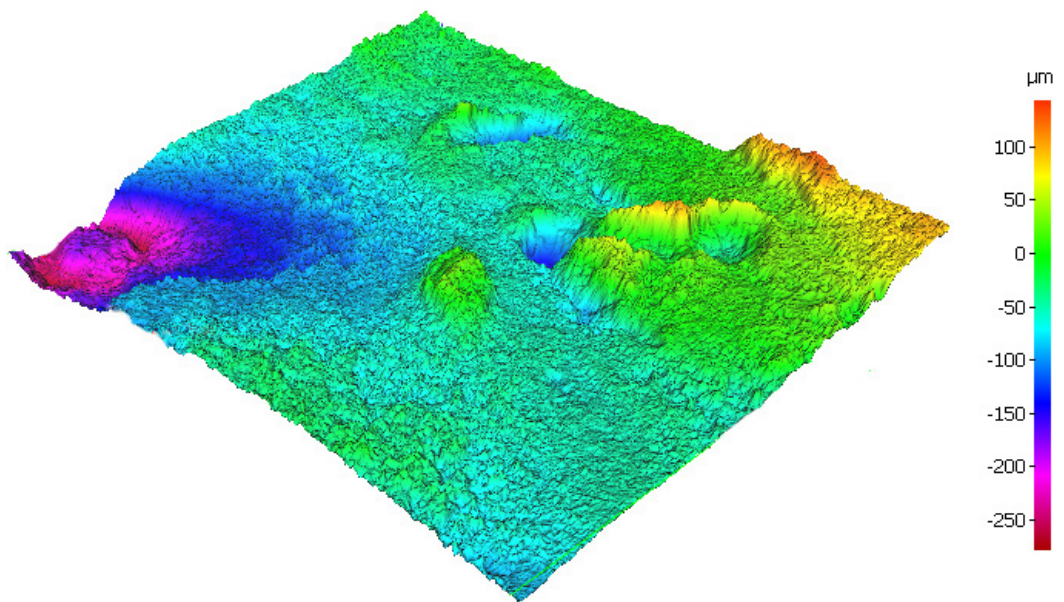

Figura 6. Modelo tridimensional reconstruído pelo MeX para a superfície de fratura região CD10/25a.

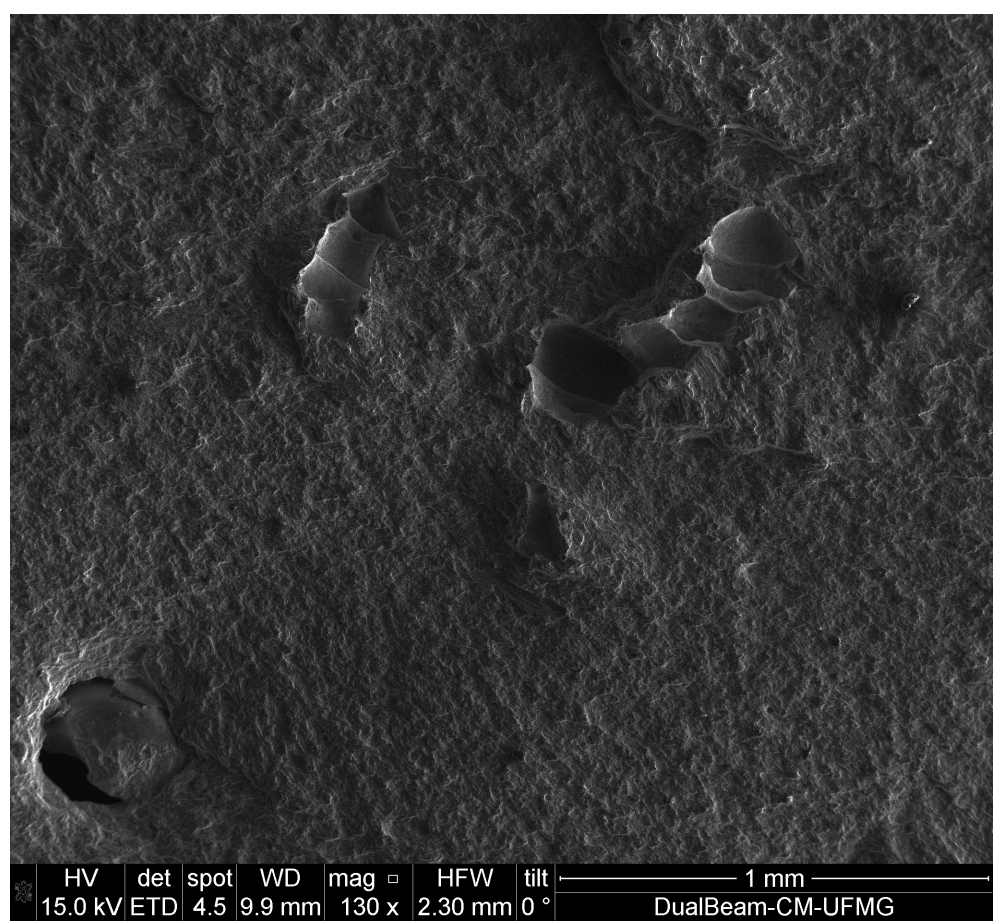

Figura 7. Superfície de fratura da região CD10/25a.

Além disso, a diferença na resolução das técnicas, tanto na altura (direção $z$ ) como no plano (direções $x$ e y) também pode ser uma fonte adicional dessas diferenças nos resultados. As resoluções lateral e vertical da estereofotogrametria são limitadas pela resolução do MEV e pela correta coleta das imagens (com relação ao requisito eucêntrico e à qualidade das imagens, por exemplo) e manipulação no software (configuração do offset, por exemplo).

De maneira geral, os resultados obtidos pelo MeX são condizentes com os da perfilometria, com diferenças importantes a serem consideradas para a coordenada z, o que resulta em algumas das discrepâncias encontradas para os parâmetros de rugosidade calculados por ambas as técnicas. Nesse sentido, acredita-se que mais testes devam ser executados a fim de se determinar as melhores condições para a coleta das imagens para a estereofotogrametria, principalmente com relação ao ângulo de inclinação mais adequado para a topografia das superfícies analisadas. 

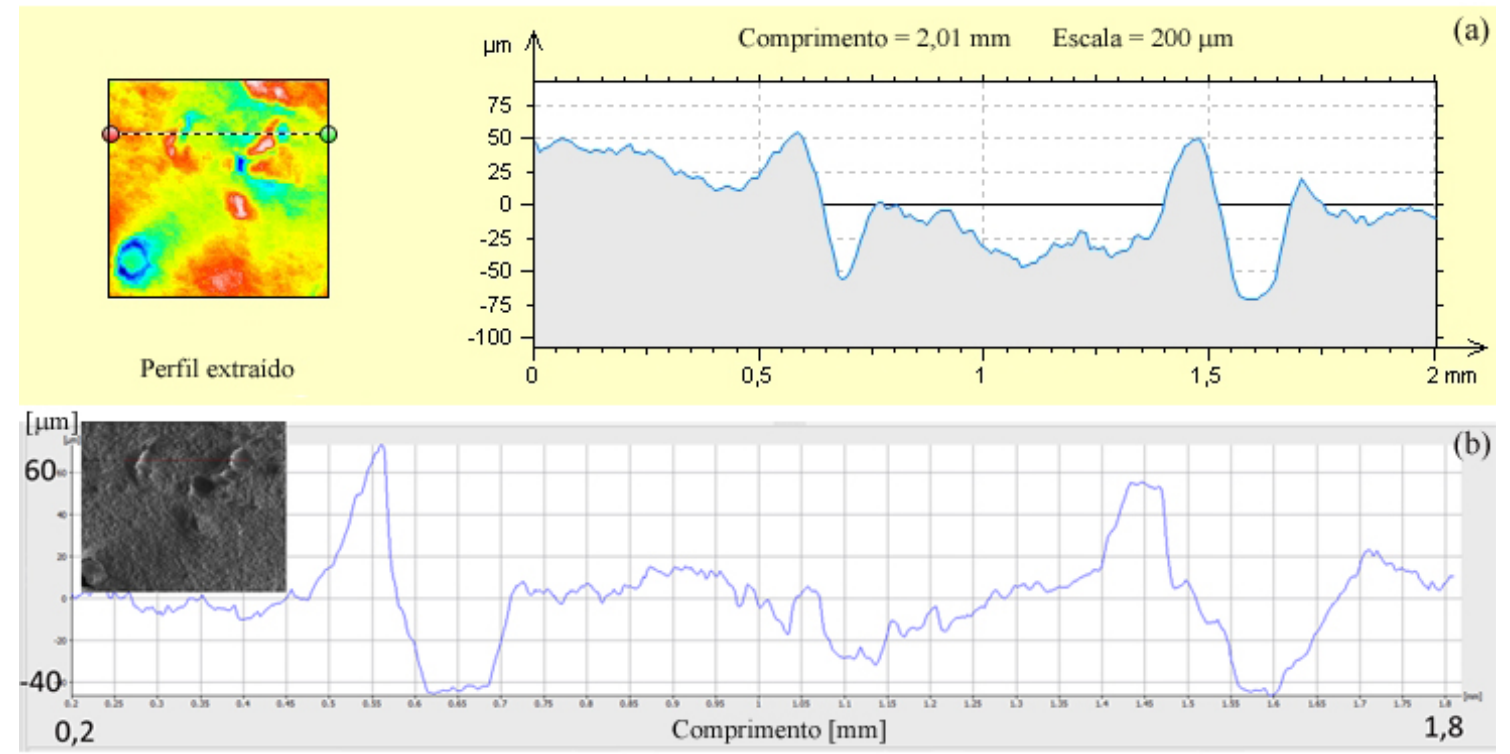

Figura 8. Referências para a obtenção dos perfis de rugosidade para extração dos parâmetros para a região CD10/25a: (a) perfilômetro de contato; (b) MeX.

Tabela 4. Parâmetros de rugosidade obtidos para a superfície da região CD10/25a pelas técnicas de perfilometria de contato e estereofotogrametria.

\begin{tabular}{ccc}
\hline $\begin{array}{c}\text { Parâmetros de } \\
\text { rugosidade }[\boldsymbol{\mu} \boldsymbol{m}]\end{array}$ & Perfilometria & MeX \\
$\mathrm{Ra}$ & 14,4 & 15,6 \\
$\mathrm{Rq}$ & 19,7 & 22,3 \\
$\mathrm{Rz}$ & 99,6 & 70,5 \\
$\mathrm{Sa}$ & 24,5 & 10,4 \\
$\mathrm{Sq}$ & 31,0 & 16,4 \\
$\mathrm{Sz}$ & 239,0 & 219,7 \\
\hline
\end{tabular}

\subsection{Caracterização das superfícies reconstruídas}

A primeira etapa para se caracterizar as superfícies de fratura consistiu em identificar os parâmetros 3D de amplitude Sa e Sq e a razão entre as áreas medida e projetada como tendo melhores relações com os vários níveis de $\Delta K$, conforme ilustra a Figura 9a-c. Os demais parâmetros de rugosidade estudados ( $R a, R q, R z$ e $S z$ ) não mostraram tendências claras com os valores de $\Delta K$. Estes dados foram colocados em função da profundidade em que a solda foi produzida, tendo em vista que foi observado que o método do ensaio (redução de carga ou carga constante) não afeta os resultados. Os parâmetros de rugosidade foram obtidos após filtragem dos comprimentos de onda superior a 0,8 $\mathrm{mm}$ a partir do filtro cut-off.

A partir da avaliação desses dados, observa-se que com o aumento do $\Delta K$ a superfície torna-se mais rugosa. Entretanto, deve-se considerar juntamente a esse, o efeito da quantidade de poros amostrada em cada posição.

A caracterização do comportamento da trinca de fadiga para as soldas subaquáticas obtidas nas profundidades de 10 e $90 \mathrm{~m}$ foi conduzida pela avaliação dos dados de rugosidade das superfícies geradas, bem como pela observação dos modelos tridimensionais reconstruídos pelo MeX. Observa-se que nas posições em que foi amostrada maior quantidade de poros as deflexões das trincas são mais acentuadas com a consequente interação da trinca com os poros. Este resultado é corroborado pelos valores de rugosidade maiores apresentados pelas superfícies em que mais poros foram amostrados, conforme ilustrado na Figura $9 \mathrm{~d}$. Além da quantidade, o tamanho dos poros também intensifica esse efeito. 

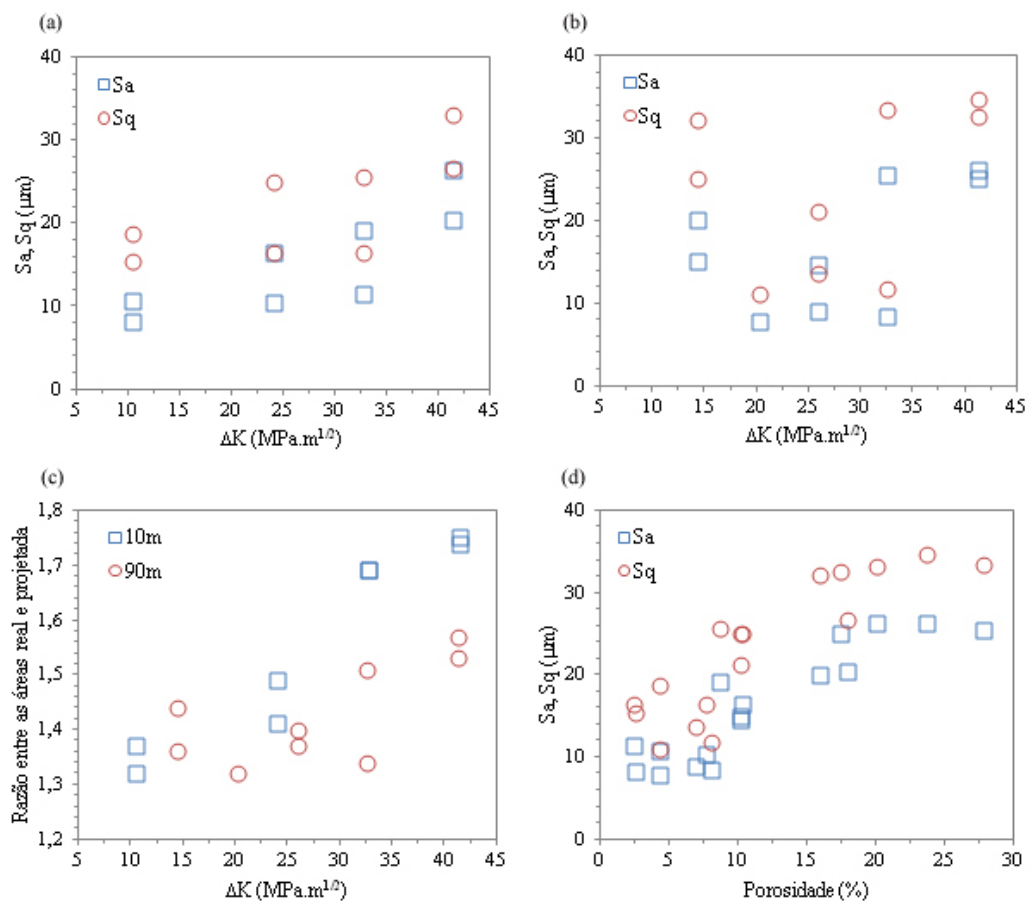

Figura 9. Relação entre os parâmetros (a); (b) Sa e Sq com para as superfícies avaliadas das soldas nas profundidades de 10 e 90 m, respectivamente; (c) razão entre as áreas medida e projetada e $\Delta K$; e (d) Sa e Sq e a quantidade de poros presente nas regiões avaliadas.

É importante ressaltar que esse resultado é válido independente da profundidade de soldagem, pois como apenas algumas regiões das superfícies dos corpos de prova selecionadas foram avaliadas e a escolha dessas regiões foi baseada unicamente nos valores de $\Delta K$ apresentados, ocorreu que algumas regiões dos corpos de prova CC10 e CD10 soldados a 10 m apresentam maior porosidade que algumas dos corpos de prova CC90 e CD90 soldados a maior profundidade, conforme mostrado na Tabela 3.

Análise de regressão linear múltipla em um programa estatístico (Minitab) foi conduzida. A partir dos resultados obtidos (Tabela 5), é possível verificar que os valores Sa e Sq são significativamente afetados pela porosidade, enquanto a razão entre as áreas medida e projetada é influenciada pela profundidade e $\Delta \mathrm{K}$, considerando uma significância menor que 5\%.

A dimensão fractal das superfícies reconstruídas também foi obtida com o intuito de correlacioná-la com as características dinâmicas da falha por fadiga. A Figura 10a compara os valores de dimensão fractal, extraídos dos modelos reconstruídos com ângulo de inclinação $\pm 5^{\circ}$ e cut-off de $800 \mu \mathrm{m}$, para as superfícies reconstruídas. Nota-se que os valores de dimensão fractal aumentaram a partir do $\Delta \mathrm{K}$ igual a $30 \mathrm{MPa} \cdot \mathrm{m}^{1 / 2}$. Além disso, o comportamento da dimensão fractal em relação ao $\Delta \mathrm{K}$ assemelha-se muito com a sua relação com o tamanho da trinca (Figura 10b).

Tabela 5. Análise de regressão linear múltipla.

\begin{tabular}{|c|c|c|c|c|c|c|}
\hline \multirow{2}{*}{ Parâmetros } & \multirow{2}{*}{ Variáveis } & \multicolumn{2}{|c|}{$\begin{array}{c}\text { Coeficientes não } \\
\text { padronizados }\end{array}$} & \multirow{2}{*}{$\begin{array}{c}\begin{array}{c}\text { Coeficientes } \\
\text { padronizados }\end{array} \\
\text { Beta }\end{array}$} & \multirow[t]{2}{*}{$\mathbf{t}$} & \multirow{2}{*}{ Sig. } \\
\hline & & B & Erro Padrão & & & \\
\hline Sa & Porosidade & 0,91 & 0,15 & 0,91 & 6,02 & 0,000 \\
\hline $\mathrm{Sq}$ & Porosidade & 1,03 & 0,18 & 1,03 & 5,62 & 0,000 \\
\hline \multirow[t]{2}{*}{ Razão } & Profundidade & $-0,54$ & 0,15 & $-0,54$ & $-3,51$ & 0,004 \\
\hline & $\Delta \mathrm{K}$ & 0,64 & 0,17 & 0,64 & 3,72 & 0,003 \\
\hline \multirow[t]{2}{*}{ Df } & Profundidade & $-0,46$ & 0,17 & $-0,46$ & $-2,73$ & 0,018 \\
\hline & $\Delta \mathrm{K}$ & 0,79 & 0,19 & 0,79 & 4,15 & 0,001 \\
\hline
\end{tabular}



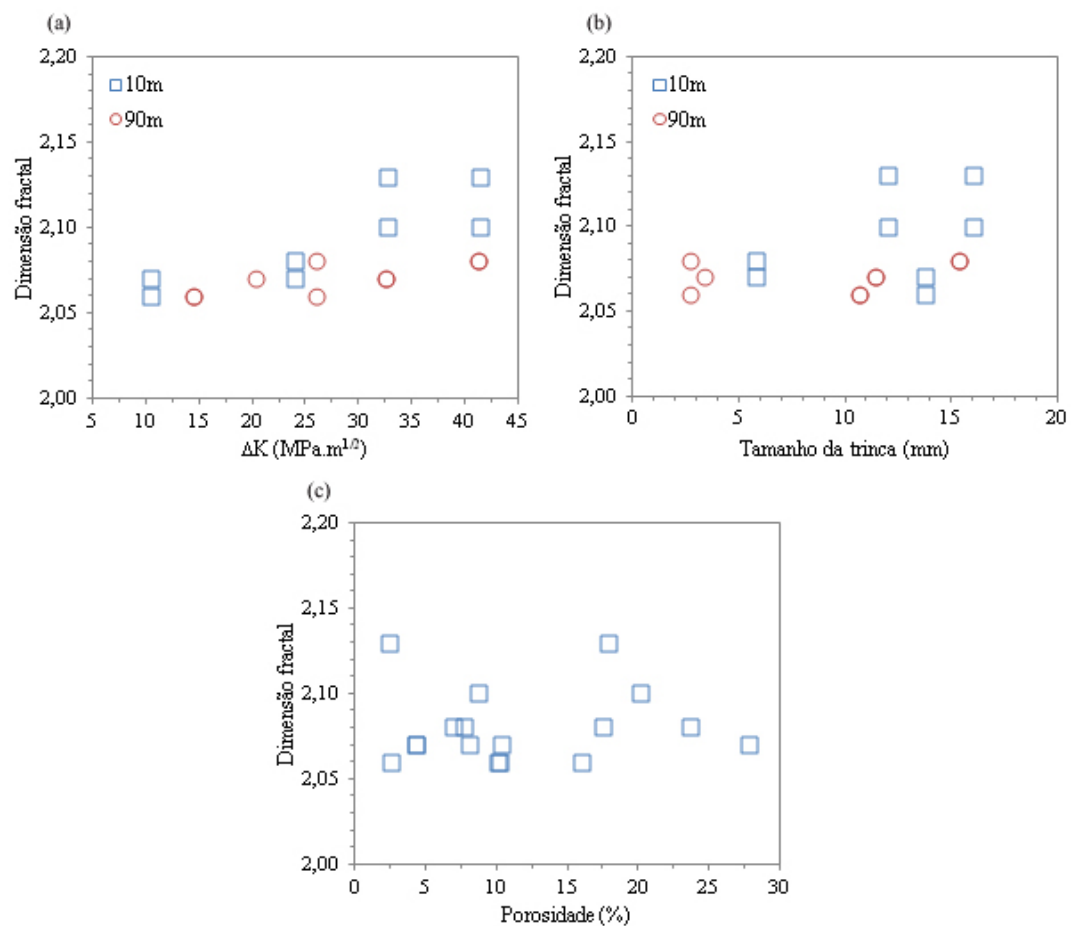

Figura 10. Relação entre a dimensão fractal e (a) $\Delta K$; (b) tamanho de trinca; e (c) porosidade.

Esse resultado já era esperado uma vez que a taxa de propagação da trinca depende de $\Delta K$. Desse modo, à medida que a velocidade de propagação da trinca aumenta, os valores da dimensão fractal, associados às respostas do material às condições mecânicas locais do processo de fratura, aumentam.

É interessante notar que tanto a região II quanto a região do limiar estudadas apresentam praticamente a mesma dimensão fractal, apesar das diferenças de condições de ensaio. Esse fato pode indicar que essas superfícies foram formadas por um mecanismo de fratura em comum, conforme relatado no estudo de Tanaka et al. [29]. Esses autores reportam ainda que a fratura ocorre pelo mecanismo de escorregamento.

Além disso, a homogeneidade da dimensão fractal para as superfícies estudadas pode inviabilizar, por outro lado, a utilização deste parâmetro para caracterizar e comparar tais superfícies. Portanto, a descrição de rugosidade das superfícies pode ser feita de modo mais eficiente, para este caso em estudo, adotando os parâmetros de textura superficial, especialmente Sq.

Em adição, a partir da Figura 10c, que mostra a relação entre a dimensão fractal e a porosidade nas regiões analisadas, é possível verificar que a presença de poros não afeta estes resultados, ao contrário do que foi observado para os parâmetros de rugosidade Sa e Sq (Figura 9d). Esse resultado é corroborado pela análise estatística realizada com significância menor que $5 \%$. De acordo com essa análise, a dimensão fractal é influenciada apenas pela profundidade e $\Delta \mathrm{K}$, conforme mostra a Tabela 5 .

Já com relação aos valores encontrados, pode-se considerar que estão condizentes com a literatura. Segundo Paggi e Carpinteri [30], a dimensão fractal não deve ser maior que 2,5 para superfícies de fratura por fadiga, uma vez que isso implicaria em características superficiais que não condizem com a dinâmica deste processo de fratura.

Finalmente, considerando a metodologia adotada para o estudo de superfícies de fratura, foi possível obter modelos tridimensionais que reproduzem a topografia das superfícies e também obter algumas conclusões no que se refere ao emprego dos parâmetros de rugosidade e dimensão fractal na caracterização das superfícies estudadas. Por outro lado, estudos mais aprofundados para compreender melhor os mecanismos de propagação de trinca nestes corpos de provas se fazem necessários, tanto com relação à aplicação do software MeX quanto à avaliação em si dos resultados encontrados por esse método. No primeiro caso, acredita-se ser necessário investigar 
mais profundamente a influência de parâmetros como distância de trabalho no MEV e ângulo de inclinação para obtenção das imagens, bem como definir condições para obtenção das imagens com qualidade ótima para o processamento do software. Já para a análise dos resultados, os valores de rugosidade obtidos podem estar influenciados pela presença desses poros (variações de altura associadas a eles), já que estes são considerados nessas medidas. Dessa forma, considera-se importante avaliar o efeito da presença de porosidade nas medições de rugosidade e buscar formas para eliminá-lo ou minimizá-lo. Além disso, um mapeamento mais extenso das superfícies, obtendo mais regiões ao longo da superfície para análise, pode fornecer mais informações a respeito do papel dos parâmetros de rugosidade e dimensão fractal para a caracterização de tais superfícies.

\section{Conclusões}

Superfícies de fratura geradas por fadiga de soldas subaquáticas molhadas foram avaliadas com relação à profundidade de soldagem e às condições de carregamento durante ensaios de fadiga. A geometria do caminho das trincas e a topografia das superfícies foram estudadas por meio de reconstrução tridimensional da superfície de fratura usando estereofotogrametria via MEV. Análise quantitativa das superfícies tridimensionais reconstruídas foi conduzida, focando principalmente na análise de perfil, parâmetros de rugosidade e dimensão fractal. As principais conclusões podem ser resumidas como se segue:

- A aplicação do software MeX mostrou-se como um método conveniente para a reconstrução tridimensional de superfícies a partir de trios de imagens estereoscópicas;

- A partir da comparação entre os métodos de perfilometria de contato e estereofotogrametria, verificou-se diferenças consideráveis para a coordenada $z$ dos modelos reconstruídos, resultando em discrepâncias dos parâmetros de rugosidade. Os parâmetros Ra e Rq apresentaram as menores diferenças na ordem de 10\%;

- Com o aumento do $\Delta K$ observou-se aumento da rugosidade das superfícies. Resultado semelhante foi encontrado para o aumento da quantidade de poros. Neste caso, a razão para isto pode estar no caminho mais tortuoso da trinca devido a sua interação com os poros ou na própria presença dos poros que contribuem para a obtenção de valores mais elevados dos parâmetros;

- As superfícies de fratura exibem comportamento fractal homogêneo com dimensão fractal no intervalo de 2,06 a 2,12, para as condições de carregamento estudadas, aumentando para maiores valores de $\Delta K$. Devido ao pequeno intervalo em que este parâmetro se encontra, acredita-se que o parâmetro de rugosidade Sq possa ser melhor empregado para caracterizar e comparar essas superfícies. Além disso, a porosidade não demonstrou afetar os valores de dimensão fractal;

- Por fim, o procedimento proposto para estudar as superfícies de fratura geradas por fadiga em soldas subaquáticas molhadas consistindo na avaliação com as técnicas de reconstrução selecionadas e análise quantitativa para determinar relações com os resultados dos ensaios de crescimento de trinca por fadiga, bem como com a profundidade de soldagem foi bem sucedida no que se refere a estabelecer algumas correlações entre estes. Entretanto, ainda são necessários estudos mais aprofundados para compreender melhor os mecanismos de propagação de trinca nestes corpos de provas.

\section{Agradecimentos}

Os autores agradecem pelo suporte recebido do Centro de Microscopia e do Laboratório de Superfícies da UFMG. Agradecemos também a CAPES-PROEX, CNPq e FAPEMIG.

\section{Referências}

[1] Pessoa ECP, Bracarense AQ, Zica EM, Liu S, Perez-Guerrero F. Porosity variation along multipass underwater wet welds and its influence on mechanical properties. Journal of Materials Processing Technology. 2006;179(1-3):239-243. http://dx.doi. org/10.1016/j.jmatprotec.2006.03.071.

[2] Buffière J-Y, Savelli $S$, Jouneau $P H$, Maire E, Fougères R. Experimental study of porosity and its relation to fatigue mechanisms of model Al-Si7-Mg0.3 cast Al alloys. Materials Science and Engineering. 2001;316(1-2):115-126. http://dx.doi. org/10.1016/S0921-5093(01)01225-4.

[3] Yan Y, Nash G, Nash P. Effect of density and pore morphology on fatigue properties of sintered Ti-6Al-4V. International Journal of Fatigue. 2013;55:81-91. http://dx.doi.org/10.1016/j. ijfatigue.2013.05.015. 
[4] Torralba J, Esteban L, Bernardo E, Campos M. Understanding contribution of microstructure to fracture behaviour of sintered steels. Powder Metallurgy. 2014;57(5):357-364. http://dx.doi. org/10.1179/1743290114Y.0000000119.

[5] Wu SC, Yu C, Zhang WH, Fu YN, Helfen L. Porosity induced fatigue damage of laser welded 7075-T6 joints investigated via synchrotron X-ray microtomography. Science and Technology of Welding and Joining. 2015;20(1):11-19. http://dx.doi.org/10 $.1179 / 1362171814$ Y.0000000249.

[6] Ferrié E, Buffière J-Y, Ludwig W. 3D characterisation of the nucleation of a short fatigue crack at a pore in a cast Al alloy using high resolution synchrotron microtomography. International Journal of Fatigue. 2005;27(10-12):1215-1220. http://dx.doi. org/10.1016/j.ijfatigue.2005.07.015.

[7] Deng X, Piotrowski G, Chawla N, Narasimhan KS. Fatigue crack growth behavior of hybrid and prealloyed sintered steels. Materials Science and Engineering. 2008;491(1-2):19-27. http:// dx.doi.org/10.1016/j.msea.2008.05.009.

[8] Arias AR, Bracarense AQ. Velocidade de propagação de trinca por faiga de soldas subaquáticas molhadas: avaliação fora da água. Soldagem e Inspeção. 2015;20(4):403-411. http://dx.doi. org/10.1590/0104-9224/SI2004.07.

[9] Arias A, Bracarense A. Fatigue crack growth assessment in underwater wet welds. Welding Journal. 2017;96:287-294.

[10] Arias A. Avaliação da propagação de trinca por fadiga em soldas subaquáticas molhadas [tese de doutorado]. Belo Horizonte: Universidade Federal de Minas Gerais; 2017. 96 p.

[11] Matlock D, Edwards GR, Olson DL, Ibarra S. Effect of sea water on the fatigue crack propagation characteristics of welds for offshore structures. Journal of Materials Engineering. 1987;9(1):25-34. http://dx.doi.org/10.1007/BF02833784.

[12] Stampfl J, Scherer S, Gruber M, Kolednik O. Reconstruction of surface topographies by scanning electron microscopy for application in fracture research. Applied Physics A: Materials Science \& Processing. 1996;63(4):341-346. http://dx.doi. org/10.1007/BF01567324.

[13] Cavallini M, Di Bartolomeo O, lacoviello F. Fatigue crack propagation damaging micromechanisms in ductile cast irons. Engineering Fracture Mechanics. 2008;75(3-4):694-704. http:// dx.doi.org/10.1016/j.engfracmech.2007.02.002.

[14] Vojtek T, Pokluda J, Hohenwarter A, Pippan R. Three-dimensional morphology of fracture surfaces generated by modes II and III fatigue loading in ferrite and austenite. Engineering Fracture Mechanics. 2013;108:285-293. http://dx.doi.org/10.1016/j. engfracmech.2013.02.022.

[15] Carli L. 3D-SEM metrology for coordinate measurements at the nanometer scale. Kongens Lyngby: Technical University of Denmark; 2010. 252 p.

[16] Gadelmawla E, Koura MM, Maksoud TMA, Elewa IM, Soliman $\mathrm{HH}$. Roughness parameters. Journal of Materials Processing Technology. 2002;123(1):133-145. http://dx.doi.org/10.1016/ S0924-0136(02)00060-2.

[17] Blunt L, Jiang X. Numerical parameters for characterisation of topography. In: Blunt L, Jiang X. Advanced techniques for assessment surface topography: development of a basis for 3D surface texture standards "surfstand". West Sussex: Kogan
Page Science; 2003. cap. 2, p. 17-41. http://dx.doi.org/10.1016/ B978-190399611-9/50002-5.

[18] Blunt L. Introduction: the history and current state of 3D surface characterisation. In: Blunt L, Jiang X. Advanced techniques for assessment surface topography: development of a basis for 3D surface texture standards "surfstand". West Sussex: Kogan Page Science; 2003. cap. 1, p. 1-13.

[19] Ohlsson R, Rosén B, Westberg J. The interrelationship of 3D surface characterisation techiques with standardised 2D techniques. In: Blunt L, Jiang X. Advanced techniques for assessment surface topography: development of a basis for 3D surface texture standards "surfstand". West Sussex: Kogan Page Science; 2003. cap. 8, p. 197-220.

[20] Bhushan B. Surface roughness analysis and measurement techniques. In: Bhushan B. Modern tribology handbook. Vol. 1. Boca Raton: CRC Press; 2000. cap. 2. http://dx.doi. org/10.1201/9780849377877.ch2.

[21] Silva EM. Análise da superfície de fratura do aço inoxídavel duplex UNS S31803 através da aplicação da geometria dos fractais [dissertação]. Fortaleza: Universidade Federal do Ceará; 2002.

[22] Oliveira JA. Comportamento fractal de superfícies de fadiga em ligas aeronáuticas [monografia]. Guaratinguetá: Faculdade de Engenharia de Guaratinguetá, Universidade Estadual Paulista; 2012.

[23] Cherepanov GP, Balankin AS, Ivanova VS. Fractal fracture mechanics: a review. Engineering Fracture Mechanics. 1995;51(6):997-1033. http://dx.doi.org/10.1016/0013-7944(94)00323-A.

[24] Pippan R, Hohenwarter A. Fatigue crack closure: a review of the physical phenomena. Fatigue \& Fracture of Engineering Materials \& Structures. 2017;40(4):471-495. http://dx.doi. org/10.1111/ffe.12578. PMid:28616624.

[25] Jha S, Szczepanski CJ, Golden PJ, Porter WJ 3rd, John R. Characterization of fatigue crack-initiation facets in relation to lifetime variability in Ti-6Al-4V. International Journal of Fatigue. 2012;42:248-257. http://dx.doi.org/10.1016/j.ijfatigue.2011.11.017.

[26] Mbuya T, Sinclair I, Moffat AJ, Reed PAS. Micromechanisms of fatigue crack growth in cast aluminium piston alloys. International Journal of Fatigue. 2012;42:227-237. http://dx.doi.org/10.1016/j. ijfatigue.2011.10.015.

[27] Alicona. MEX version 6.1. Austria: Alicona Imaging GmbH; 2013.

[28] Watson P, Tsai C, Wood B. Fitness for service design application for underwater wet welds. In: Liu S. Proceedings of the International Workshop on Underwater Welding of Marine Structures; 1994; New Orleans. New York: American Bureau of Shipping; 1994. p. 215-251.

[29] Tanaka M, Kimura Y, Taguchi J, Kato R. Fracture surface topography and fracture mechanism in austenitic SUS316 steel plates fatigued by repeated bending. Journal of Materials Science. 2006;41(10):2885-2893. http://dx.doi.org/10.1007/ s10853-005-5160-0.

[30] Paggi M, Carpinteri A. Fractal and multifractal approaches for the analysis of crack-size dependent scaling laws in fatigue. Chaos, Solitons, and Fractals. 2009;40(3):1136-1145. http:// dx.doi.org/10.1016/j.chaos.2007.08.068. 
Soldagem \& Inspeção. 2018;23(4):515-515

https://doi.org/10.1590/0104-9224/SI2304.10

ISSN 1980-6973 (Online)

ISSN 0104-9224 (Print)

\section{Errata}

No artigo Caracterização por Reconstrução Tridimensional da Superfície de Fratura Gerada por Fadiga em Soldas Subaquáticas Molhadas, com número de DOI: https://doi.org/10.1590/0104-9224/SI2302.03, publicado no periódico Revista Soldagem \& Inspeção, 23 (2): 143-156, na página 143:

Onde se lia:

"Ariel Rodrigues Arias"

Leia-se:

"Ariel Rodriguez Arias" 